\title{
Efeito do exercício aquático terapêutico em mulheres com osteoartrose de joelho: um estudo randomizado controlado
}

\author{
Effect of therapeutic aquatic exercise in \\ women associated with knee osteoarthrosis:
}

a randomized controlled trial

FisiSenectus . Unochapecó Ano 5, n. 1 - Jan/Jun. 2017 p. $31-41$

Luana Meneghini Belmonte. Iuana.meneghini@unisul.br

Fisioterapeuta. Docente do Curso de Fisioterapia da Universidade do Sul de Santa Catarina - UNISUL.

Priscila Martins Gerent. prigerent@hotmail.com Fisioterapeuta.

Fabiane Zschornak da Silva. fabe_zschornak@hotmail.com Fisioterapeuta.

Inês Alessandra Xavier de Lima. inesaxlima1@gmail.com Fisioterapeuta. Docente do Curso de Fisioterapia da Universidade do Sul de Santa Catarina - UNISUL. Programa de Pós-Graduação em Engenharia de Produção da Universidade Federal de Santa Catarina - PPGEP/UFSC.

Luiz Augusto Oliveira Belmonte. Iuiz.belmonte@unisul.br Fisioterapeuta. Docente do Curso de Fisioterapia da Universidade do Sul de Santa Catarina - UNISUL. Programa de Pós-Graduação em Ciências da Saúde da Universidade do Sul de Santa Catarina - PPGCS/UNISUL.

\section{Resumo}

Introdução: A Osteoartrose $(\mathrm{OA})$ é uma doença crônico-degenerativa, que afeta as articulações sinoviais, sendo o joelho a mais afetada. Caracteriza-se por dor, rigidez articular, perda de força muscular, influenciando na funcionalidade. 0 exercício terapêutico aquático apresenta-se como uma das principais intervenções terapêuticas no tratamento da OA. Objetivo: Analisar os efeitos do exercício terapêutico aquático na dor, aptidão física e funcionalidade de joelho de mulheres com diagnóstico clínico de osteoartrose de joelho. Materiais e métodos: Estudo do tipo Ensaio Clínico Randomizado. A amostra foi composta por 28 indivíduos, separados em dois grupos: I ( $n=15$; intervenção) e C ( $n=13$; controle). Os instrumentos utilizados para coleta dos dados foram: Escala Visual Analógica, Bateria (de teste) Senior Fitness Test e Questionário Lysholm. Resultados: Houve diferença significativa no grupo intervenção nas variáveis: dor (avaliação=7,43 $( \pm 1,8)$; reavaliação $5,47( \pm 3,2) ; p=0,005)$, função do joelho (avaliação=30,6 $( \pm 17,4)$; reavaliação $48,2( \pm 19,7)$; $p=0,005)$, levantar da cadeira (avaliação=5,6 $( \pm 2)$; reavaliação $7,4( \pm 2,1) ; p=0,005)$, flexão do braço (avaliação=10 $( \pm 3,5)$; reavaliação $14( \pm 3,3) ; \quad p=0,001)$ e resistência aeróbica (avaliação=327,6 $( \pm 129)$; reavaliação $382,2( \pm 115) ; p=0,000)$, enquanto no grupo controle

\section{Fisißenectus}


somente na dor (avaliação=7,92 $( \pm 1,8)$; reavaliação $6,46( \pm 3,2) ; p=0,013)$. Conclusão: A intervenção com exercícios terapêuticos aquáticos foi eficaz em relação à melhora da dor, função do joelho, força muscular e resistência aeróbica.

\title{
Palavras-chave
}

Osteoartrose; Hidroterapia; Dor; Aptidão física.

\begin{abstract}
Introduction: Osteoarthrosis $(\mathrm{OA})$ is a chronic degenerative disease that affects synovial articulation, being knee the most affected. Consisting of Pain, joint stiffness, loss of muscle strength, it influences the functionality. Aquatic therapy exercise is shown as one of the main interventions in the OA treatment. Objective: to analyze the effects of the aquatic therapy exercise in pain, fitness and knee functionality in women with clinical diagnosis osteoarthrosis knee. Materials and methods: it was a Randomized Clinical Paired Trial. The sample was composed of 28 individuals, divided in two groups: A ( $n=15$; intervention) and B ( $n=13$; control). The instruments used to collect data were Visual Analog Scale, Senior Fitness Test and Lysholm Questionnaire. Results: there was significant difference in the intervention group in the following variables: pain (evaluation $=7,43( \pm 1,8)$; revaluation $5,47( \pm 3,2) ; p=0,005)$, knee function (evaluation=30,6 $( \pm 17,4)$; revaluation $48,2( \pm 19,7)$; $p=0,005)$, to get up from the chair (evaluation $=5,6( \pm 2)$; revaluation 7,4 $( \pm 2,1) ; p=0,005)$, arm flexion (evaluation $=10( \pm 3,5)$; reavaluation $14( \pm 3,3) ; p=0,001$ ) and aerobic resistance (evaluation=327,6 $( \pm 129)$; revaluation $382,2( \pm 115) ; p=0,000)$, while in control group only pain it was verified (evaluation $=7,92( \pm 1,8)$; revaluation $6,46( \pm 3,2) ; p=0,013)$. Conclusion: Aquatic therapy exercise intervention was effective regarding to pain improvement, knee function, muscle strength and aerobic resistance.
\end{abstract}

\section{Keywords}

Osteoarthrosis; Arthrosis; Hydrotherapy; Pain; Physical fitness.

\section{Introdução}

$\infty \infty \infty \times \infty \times \infty \times \infty \times \infty \times \infty \times \infty \times \infty \times \infty \times \infty \times \infty \times \infty$

A osteoartrose $(O A)$ é a doença crônica que leva à dor e disfunção na vida adulta mais frequente e prevalente no mundo, sendo que as articulações mais acometidas são o quadril e joelho. Cerca de $70 \%$ a $80 \%$ da população com mais de 55 anos têm alterações degenerativas nas articulações e a OA é encontrada em $33 \%$ da população com mais de 60 anos de idade ${ }^{1,2}$. A OA é responsável por uma maior incidência de deficiência do que qualquer outra doença crônica ${ }^{3}$. Apresenta uma fisiopatologia ainda não compreendida completamente, que consiste em um processo de inflamação e degeneração associado à degeneração da cartilagem articular, hipertrofia nas margens óssea e alterações na membrana sinovial. Os sintomas típicos da OA incluem início gradual de dor e rigidez em torno de uma articulação com a diminuição da função ${ }^{4,5}$. A OA dos membros inferiores pode afetar seriamente a independência, estilo de vida e o cuidado em pessoas idosas ${ }^{6}$. Neste contexto, a OA de joelho pode gerar uma redução da atividade funcional e pode levar a uma fraqueza muscular adicional com alteração da nutrição da cartilagem, contribuindo para a manutenção das deficiências, limitações funcionais e aumento da incapacidade ${ }^{7}$.

As mulheres geralmente estão mais expostas aos efeitos da degeneração articular no joelho pelo fato de apresentar menor cartilagem articular na porção proximal da tíbia e da patela. Acredita-se que essa prevalência também se deve ao fator hormonal ${ }^{8,9}$.

Não há, atualmente, um tratamento único considerado mais eficaz para a OA. O tratamento pode ser constituído por diversas modalidades, como exercício físico, educação, fisioterapia, perda de peso e farmacológicos (com medicamentos anti-inflamatórios não-esteróides, acetaminofeno, glucosamina, condroitina e cirúrgico ${ }^{10}$. Estas modalidades terapêuticas não tem efeito de reversão ou prevenção em relação às alterações bioquímicas na cartilagem, portanto, a gestão atual da OA centra-se na redução dos sintomas e melhora da 
função, sendo o exercício físico, independentemente da modalidade ou tipo (força ou aeróbica), o recurso terapêutico que tem se mostrado eficaz em atingir estes objetivos ${ }^{10}$.

Os exercícios terapêuticos aquáticos (ETAs) se destacam como uma das principais intervenções terapêuticas no tratamento da OA, pois as propriedades físicas da água facilitam a execução dos exercícios com intuito de reduzir o impacto articular na região afetada, tornando o exercício menos doloroso ${ }^{11}$. 0 calor da água diminui a sensibilidade das fibras nervosas rápidas (tato) e lentas (dor), liberando endorfinas que diminuem a sensação de dor, promovendo relaxamento muscular e, consequentemente, melhorando a mobilidade articular ${ }^{12}$.

Há necessidade de estudos buscando o entendimento dos sintomas da OA e de estratégias que contribuam para a promoção da saúde de indivíduos portadores dessa doença, a fim de integrá-los a programas de reabilitação para melhora da capacidade funcional ${ }^{13,14}$.

Assim, o objetivo deste estudo foi analisar os efeitos dos exercícios terapêuticos aquáticos na dor, aptidão física e funcionalidade de joelho de mulheres com diagnóstico clínico de osteoartrose de joelho.

\section{Materiais e métodos}

$\infty \times \infty \times \infty \times \infty \times \infty \times \infty \times \infty \times \infty \times \infty \times \infty \times \infty \times \infty)$

Este estudo, do tipo Ensaio Clínico Randomizado (ECR) foi aprovado pelo Comitê de Ética em Pesquisa da Universidade do Sul de Santa Catarina (Unisul) sob parecer 1.400.772.

A amostra, não probabilística do tipo intencional, foi composta por 28 indivíduos do sexo feminino, cadastrados na lista de espera da Clínica Escola de Fisioterapia da UNISUL. Os critérios de inclusão foram: gênero feminino; idade igual ou superior a 50 anos; apresentar diagnóstico clínico de osteoartrose de joelho e atestado médico de liberação para realização de exercício físico. Os critérios de exclusão foram: ter realizado artroplastia de joeIho; apresentar doença cardiovascular não controlada; apresentar dor e/ou disfunções que impeçam a realização dos exercícios físicos propostos na intervenção fisioterapêutica.

Inicialmente, 42 indivíduos foram pré-selecionados, porém nem todos completaram o processo de coleta de dados (avaliação e reavaliação), levando a uma perda amostral de 14 indivíduos. Sendo assim, a amostra deste estudo foi constituída pelos 28 indivíduos que cumpriram todas as etapas da coleta de dados. A amostra foi alocada de forma aleatória, por sorteio, em dois grupos: $G I$ ( $n=15$; grupo intervenção) e GC ( $n=13$; grupo controle).

Na sequência, os indivíduos foram avaliados, etapa na qual foram coletados os seguintes dados: a) variável idade e as variáveis clínicas (local e intensidade da queixa principal e diagnóstico clínico); b) variável dor, utilizando a Escala Visual Analógica (EVA); c) variável funcionalidade do joelho, utilizando o Questionário Lysholm ${ }^{14}$; d) variável aptidão física, por meio da bateria de testes de aptidão física para idosos Senior Fitness Test (SFT) adaptado de Rikli e Jones (2008), a qual é composta por seis testes físicos: levantar da cadeira (força dos membros inferiores), flexão de braço (força dos membros superiores), sentar e alcançar os pés (flexibilidade dos membros inferiores), levantar e caminhar (agilidade e o equilíbrio dinâmico), alcançar as costas mão direita e esquerda (flexibilidade dos membros superiores) e caminhada de 6 minutos (resistência aeróbica).

A coleta de dados ocorreu antes e após a realização do protocolo de intervenção (avaliação e reavaliação, respectivamente). O GI foi submetido ao protocolo de intervenção com ETAs em grupo, enquanto o GC foi orientado a continuar com tratamento habitual para OA. A intervenção com ETAs em grupo foi realizada em uma piscina terapêutica com rampa de acesso, com medidas de $13 \mathrm{~m}$ de comprimento, $5 \mathrm{~m}$ de largura e $1,5 \mathrm{~m}$ de profundidade, com temperatura da água aproximada de $34^{\circ} \mathrm{C}$, localizada no Complexo Aquático da UNISUL. Cada intervenção teve duração de 1 hora, com frequência de 2 (duas) vezes por semana, durante o período de agosto a dezembro de 2015, totalizando 32 (trinta e duas) intervenções. 0 protocolo de intervenção com ETAs foi elaborado de acordo com o protocolo de Beker (2009) ${ }^{15}$, composto por quatro fases: aquecimento cardiovascular, alongamento muscular, fortalecimento muscular e 
relaxamento aquático. 0 aquecimento (com duração de 10 minutos) foi realizado com marcha em diversas direções; o alongamento (com duração de 20 minutos) foi realizado de forma analítica nos membros superiores e inferiores e de forma global (enfatizando a cadeia muscular posterior); o fortalecimento muscular (com duração de 25 minutos) foi realizado nos grupos musculares estabilizadores do joelho e o relaxamento aquático (com duração de 5 minutos) foi composto por técnicas de Ai Chi14,16,17.

Os dados foram analisados no programa estatístico SPSS -Statistical Package for Social Sciences - versão 17.0 para Windows. 0 tratamento estatístico descritivo foi realizado mediante frequência simples e porcentagens. Para verificar a normalidade dos dados foi utilizado o teste Shapiro-Wilk. Quanto a análise inferencial, para comparar as médias entre os dois grupos (GC e GI) foi utilizado o Teste $t$ para amostras independentes. Para comparar as médias da avaliação e reavaliação do mesmo grupo foi utilizado o Teste $t$ para amostras. Adotou-se um nível de significância de $5 \%$.

\section{Resultados}

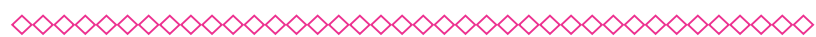

Concluíram todas as etapas do estudo 28 participantes sendo GC $(n=13)$ E GI $(n=15)$. O GC apresentou média de idade de $59( \pm 4,3)$ anos e 0 GI apresentou média de idade de $61( \pm 7,4)$ anos. Não houve diferença entre os grupos para a variável idade, o que demonstra que os grupos são homogêneos.

Nos resultados da amostra da presente pesquisa $(n=28)$ houve correlação entre as variáveis função do joelho e dor (avaliação: $r=-0,569$; $p=0,002$ e reavaliação: $r=-0,459 ; p=0,014)$, indicando que a diminuição da função do joelho está associada ao aumento da dor. Da mesma forma, verificou-se correlação entre as variáveis função do joelho e levantar da cadeira (avaliação: $r=0,393 ; p=0,039)$, indicando que a diminuição da função do joelho está associada a menor força dos membros inferiores.

A Tabela 1 apresenta os resultados da comparação entre as variáveis analisadas na avaliação das participantes do GI e GC. Nota-se que não há diferença significativa entre os grupos, confirmando que ambos são semelhantes $(p>0,05)$.

A Tabela 2 apresenta a comparação entre os resultados das variáveis do estudo no GI e no GC, na avaliação e reavaliação. Observou-se diferença significativa quando foram comparadas as médias da avaliação e reavaliação do GI das variáveis dor, função do joelho, levantar da cadeira, flexão de braço e caminhada de 6 minutos; enquanto no GC houve diferença significativa apenas na variável dor. Esses resultados indicam que a intervenção com os ETAs diminuiu a dor e melhorou a funcionalidade do joelho, força de MMII, força de MMSS e resistência aeróbica. As demais variáveis não apresentaram diferença significativa entre os grupos.

Ainda na tabela 2, quando foram comparadas as variáveis analisadas no presente estudo na reavaliação entre o GI e GC, observou-se diferença significativa na variável função do joelho (resultado final ordinal), indicando melhor função no GI.

A Figura 1 apresenta o resultado final nominal da função do joelho, na avaliação e reavaliação de cada participante do GI e do GC. Os resultados demonstram que a intervenção através dos ETAs gerou efeitos positivos, diminuindo as limitações funcionais decorrentes da OA em 6 participantes, os quais apresentaram resultado final nominal "ruim" na avaliação e "regular" na reavaliação.

A Figura 2 apresenta os resultados relacionados a intensidade da dor no joelho, na avaliação e reavaliação, das participantes do GI e GC. Os resultados demonstram que houve diminuição da intensidade da dor em 4 participantes do estudo submetidos aos ETAs, sendo que 1 referiu intensidade "leve" e 3 participantes referiram "nenhuma dor" na reavaliação. No GC, 4 participantes também referiram diminuição da dor, entretanto a intensidade da dor alterou somente de "intensa" para "moderada" na reavaliação.

A OA é a forma mais comum de doença articular crônico-degenerativa que evidencia desgaste da cartilagem articular e formação marginal osteofitária, frequentemente associada à dor, rigidez articular, deformidade e progressiva perda da função, afetando o indivíduo em múltiplas dimensões: social, psicológica e biológica ${ }^{18}$. Estudos 
afirmam que o envelhecimento está diretamente relacionado com o surgimento das doenças crônico-degenerativas, dentre elas as doenças articulares $^{9,19}$. A idade mais comum para o surgimento das degenerações articulares é a partir dos 50 $\operatorname{anos}^{20,21}$, o que vai ao encontro dos resultados encontrados no presente estudo.

A OA é mais comum nas mulheres do que nos homens; diferentes estudos verificaram que a prevalência de osteoartrose é maior em indivíduos do sexo feminino ${ }^{8,9,22}$. Este maior acometimento pode estar associado às alterações hormonais, mais precisamente à diminuição do hormônio estrógeno (condroprotetor) a partir do período pós-menopausa9,23; outra hipótese é de que as mulheres apresentam menor cartilagem articular na porção proximal da tíbia e da patela $a^{9,23,24}$.

A OA acomete as articulações que suportam descarga de peso, dentre elas a mais acometida é o joelho. Tal articulação é essencial em diversas atividades de vida diária (AVD's) como subir e descer escadas, levantar-se de uma cadeira e permanecer em pé, caminhar, agachar e ajoelhar; por isso a escolha do SFT como instrumento de avaliação da aptidão física neste estudo. 0 acometimento dessa articulação pode gerar redução da força muscular, influenciando no equilíbrio e coordenação ${ }^{25}$.

Os resultados do presente estudo demonstram diminuição quantitativa da dor após a intervenção com ETAs em 4 participantes, embora para a maioria tenha permanecido "moderada". Este resultado vai ao encontro de estudos que apontam a eficácia dos ETAs na diminuição da dor em indivíduos com osteoartrose ${ }^{19,26}$. Os exercícios aquáticos não modificam a deterioração da cartilagem, porém, o caIor da água auxilia no relaxamento da musculatura estabilizadora da articulação, além da diminuição da tensão sobre a mesma pela flutuação, promovendo alívio da dor e atuando, desta forma, no processo de desaceleração da doença ${ }^{27}$. Estas considerações vão ao encontro do presente estudo, no qual houve diminuição da dor em ambos os grupos (GI e GC). Entretanto, no grupo submetido aos ETAs houve diminuição da média da dor e a maioria dos sujeitos teve diminuição da intensidade da dor, de "intensa" para "moderada", diferentemente do que ocorreu com o grupo controle. Diferentes estudos, os quais utilizaram outros tipos de intervenção fisioterapêutica como eletroterapia, termoterapia e cinesioterapia, também verificaram diminuição do quadro álgico em indivíduos com $0 A^{26,28,29}$.

Com relação à força muscular, estudos defendem que o exercício aumenta a força de quadríceps e parece ser melhor na recuperação articular ${ }^{23,30}$. Um estudo do tipo revisão sistemática apontou que o treinamento de resistência melhorou a força muscular, o auto-relato de dor e a função física em torno de $50-75 \%$ dos estudos pesquisados ${ }^{31}$. Outra revisão sistemática também identificou benefícios dos exercícios terapêuticos em relação à dor, qualidade de vida, equilíbrio, força muscular, rigidez articular, tempo de caminhada e aptidão física. Entretanto, houve variações quanto aos benefícios dos exercícios terapêuticos em função do tipo, tempo e frequência de tratamento ${ }^{23}$. Esses resultados corroboram os do presente estudo, os quais evidenciaram melhora no teste de caminhada, ou seja, melhora da resistência aeróbica, a qual poderia ser explicada pela diminuição da dor e/ou aumento da força muscular, potencializando a função do joelho e viabilizando a melhora do ritmo da caminhada.

Estudos apontam que a diminuição da força dos músculos relacionados com a marcha como, por exemplo, o quadríceps, faz com que os indivíduos portadores de osteoartrose diminuam seu nível de atividade física e também que há redução de força muscular em torno de $15 \%$ a cada década a partir dos 50 anos, estando associada ao enveIhecimento populacional $22,25,32$.

A progressão dos sinais e sintomas associados à osteoartrose pode prejudicar a funcionalidade do joelho, gerando consequências variadas para a função e qualidade de vida dos indivíduos ${ }^{33}$. No Brasil, a Sociedade Brasileira de Reumatologia (SBR) formulou, em 2002, um consenso para o tratamento da OA que expõe como medida não farmacológica a prática de exercícios terapêuticos - Cinesioterapia, com ênfase no fortalecimento muscular, exercício aeróbico e alongamento ${ }^{34}$.

Neste sentido, exercícios resistidos são prescritos para o aumento da força muscular dos membros, sendo bem aceitos por pacientes que realizam ETAs por considerar a execução mais 
confortável no ambiente aquático do que no solo ${ }^{18}$. Esses achados corroboram os do presente estudo, no qual foi constatado a melhora da força muscular de membros inferiores (MMII) e membros superiores (MMSS) no grupo submetido à intervenção fisioterapêutica embora os exercícios não tenham enfatizado os MMSS, percebe-se que os participantes fizeram uso dos braços para se deslocarem no ambiente aquático. Acredita-se que isto tenha influenciado no ganho de força muscular dos MMSS.

Com base nos resultados apresentados neste estudo, os ETAs assumem papel importante no que diz respeito à melhora dos sintomas e restauração da função do joelho com OA.

\section{Conclusão}

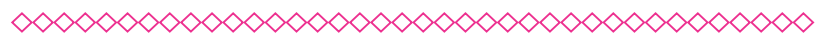

Pode-se concluir que o programa de ETAs proposto para as participantes do estudo foi eficaz em relação à melhora da dor, função do joelho, força muscular e resistência aeróbia.

Sugere-se, para avaliação funcional do joelho com OA, a utilização de questionários que considerem aspectos relacionados à dor, funcionalidade articular e atividades da vida diária (AVD's) para avaliação do estágio da doença; bem como intervenções terapêuticas que enfatizem as aptidões físicas, resultando em melhora da funcionalidade.

\section{Referências}

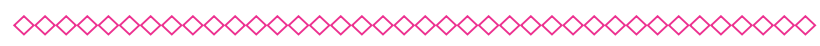

1. Lim JY, Tchai E, Jang SN. Effectiveness of Aquatic Exercise for Obese Patients with Knee Osteoarthritis: A Randomized Controlled Trial. PM\&R 2010;2(8):723-31.

2. Lu M, Su Y, Zhang Y, Zhang Z, Wang W, He Z et al. Effectiveness of aquatic exercise for treatment of knee osteoarthritis. Z Rheumatol [Internet]. 2015 Aug [acesso em 24 out 2016];74(6). Disponivel em: http://link.springer.com/10.1007/ s00393-014-1559-9
3. Bosomworth NJ. Exercise and knee osteoarthritis: benefit or hazard? Can Fam Physician [Internet]. 2009 Sep;55(9):871-8.

4. Escalante Y, García-Hermoso A, Saavedra JM. Effects of exercise on functional aerobic capacity in lower limb osteoarthritis: A systematic review. J Sci Med Sport 2011 May;14(3):190-8.

5. Batterham SI, Heywood S, Keating JL.

Systematic review and meta-analysis comparing land and aquatic exercise for people with hip or knee arthritis on function, mobility and other health outcomes. BMC Musculoskelet Disorders. BioMedCentral 2011 Jun;12:123.

6. Quintrec JL Le, Verlhac B, Cadet C, Bréville $P$, Vetel JM, Gauvain JB, et al. Physical exercise and weight loss for hip and knee osteoarthritis in very old patients: a systematic review of the literature. Open Rheumatol J [Internet]. Bentham Science Publishers 2014 [cited 2016 Oct 24]; 8:89-95. Available from: http://www.ncbi.nlm.nih.gov/ pubmed/25489352.

7. Segal NA, Wallace R. Tolerance of an aquatic power training program by older adults with symptomatic knee osteoarthritis. Arthritis [Internet]. Hindawi Publishing Corporation; 2012.

8. De Rosis RG, Kairalla M, Massabki PS. Osteoartrite: avaliação clínica e epidemiológica de pacientes idosos em instituição de longa permanência. Rev Bras Clin Med 2010;8(2)101-8.

9. Cubucku D, Sarsan A, Alkan H. Relationships between pain, function and radiographic findings in osteoarthritis of the Knee: a cross-sectional study. Arthritis. 2012;2012(April 2007):6.

10. Waller B, Ogonowska-Slodownik A, Vitor M, Lambeck J, Daly D, Kujala UM, et al. Effect of Therapeutic Aquatic Exercise on Symptoms and Function Associated With Lower Limb Osteoarthritis: Systematic Review With MetaAnalysis. Phys Ther 2014;94(10).

11. Santos MLADS, Gomes WF, Queiroz BZ De, Rosa NMDB, Pereira DS, Dias JMD, et al. Desempenho muscular, dor, rigidez e funcionalidade de idosas com osteoartrite de joelho. Acta Ortopédica Bras 2011;19(4):193-7. 
12. Barduzzi GO, Rocha Júnior PR, Souza Neto JC, Aveiro MC. Capacidade funcional de idosos com osteoartrite submetidos a fisioterapia aquática e terrestre. Fisioter Mov. [Internet] 2013;26(2): 349-60.

13. Degani AM. Hidroterapia: os efeitos físicos, fisiológicos e terapêuticos da água. Rev Fisioter Mov. 1998;6(1):91-106.

14. Loza E, Benito-Ruiz P, Blanco F, de Miguel $E$, Roman JA. Feasibility and efficacy of a multidisciplinary health care programme for patients with knee osteoarthritis. Clin Exp Rheumatol 2011;29(6):913-20.

15. Becker BE. Aquatic Therapy: Scientific Foundations and Clinical Rehabilitation Applications. PM R [Internet]. Elsevier Inc. 2009;1(9):859-72. Available from: http://dx.doi. org/10.1016/j.pmrj.2009.05.017.

16. Rikli RE, Jones CJ. Teste de aptidão física para idosos. São Paulo: Manole; 2008.

17. Cunha MCB, Alonso AC, Mesquita e Silva T, Raphael ACB, Mota CF. Ai Chi: efeitos do relaxamento aquático no desempenho funcional e qualidade de vida em idosos TT - Ai Chi: aquatic relaxing effects on functional performance and quality of life in elderly. Fisioter Mov. 2010;23(3):409-17.

18. Franciulli PM, Souza GB, Albiach JF, Santos KCP dos, Barros L de O, Santos NT dos, et al. Efetividade da hidroterapia e da cinesioterapia na reabilitação de idosos com histórico de quedas. Estud Interdiscipl Envelhec 2015;20(3):671-86.

19. Pereira RP, Amorim VM, Sandoval RA. Eficácia da hidroterapia em mulheres com osteoartrose de joelho: relato de casos. EFDeportes.com, Revista Digital. 2010.

20. Arden N, Nevitt MC. Osteoarthritis: Epidemiology. Best Pract Res Clin Rheumatol 2006;20(1):3-25.

21. Rezende MU, Gobbi RG. Tratamento medicamentoso da osteoartrose do joelho fisiopatologia da OA. Rev Bras Ortop 2009;44(1):9-14.
22. Faria PH, Faria WC, Pernambuco AP. Avaliação do nível de atividade físico e sua correlação com a dor e com o grau de comprometimento em pacientes com gonartrose. Rev Científica UNIFORMG 2014;9(1):20-30.

23. Silva A, Serrão PRMS, Driusso P, Mattiello SM. Efeito de exercícios terapêuticos no equilíbrio de mulheres com osteoartrite de joelho: Uma revisão sistemática. Brazilian J Phys Ther 2012;16(1):1-9.

24. Cicuttini F, Forbes A, Morris K, Darling S, Bailey $M$, Stuckey $S$. Gender differences in knee cartilage volume as measured by magnetic resonance imaging. Osteoarthr Cartil. W.B. Saunders 1999; 7(3):265-71.

25. Bennell KL, Hunt MA, Wrigley T V, Hunter DJ, Hinman RS. The effects of hip muscle strengthening on knee load, pain, and function in people with knee osteoarthritis: a protocol for a randomised, single-blind controlled trial. BMC Musculoskelet Disord [Internet]. BioMed Central 2007 [cited 2016 Sep 22];8:121. Available from: http://www.ncbi.nlm.nih.gov/pubmed/18067658

26. Cochrane T, Davey RC, Matthes Edwards SM. Randomised controlled trial of the costeffectivenessof water-based therapy for lower limb osteoarthritis. Health Technol Assess 2005;9(31).

27. Bates A, Hanson N. Exercícios aquáticos terapêuticos. Manole. São Paulo; 1998.

28. Silva ALP, Imoto DM, Croci AT. Estudo comparativo entre a aplicação de crioterapia, cinesioterapia e ondas curtas no tratamento da osteoartrite de joelho. Acta Ortopédica Bras 2007; 15(4):204-9

29. Baar ME Van, Dekker J, Oostendorp RAB, Bijl D, Voorn TB, Bijlsma JWJ. E V ectiveness of exercise in patients with osteoarthritis of hip or knee: nine months follow up. 2001;1123 30.

30. Wannmacher L. Osteoartrose de joelhos. Parte II: evidências sobre abordagens não medicamentosas. v. 3. 2006.

31. Lange AK, Vanwanseele $B$, Fiatarone Singh $M$. Strength training for treatment of osteoarthritis 
of the knee: a systematic review. Arthritis Rheum 2008;59(10):1488-1494.

32. Silva TAA, Frisoli Junior A, Pinheiro MM, Szejnfeld VL. Sarcopenia Associada ao envelhecimento: aspectos etiológicos e opções terapêuticas. Rev Bras Reumatol 2006;46(6): 391-7.

33. Peccin MS, Ciconelli R, Cohen M. Questionário específico para sintomas do joelho "Lysholm Knee
Scoring Scale" - Tradução e validação para a língua portugesa. Acta Ortopédica Bras 2006;14(5):3-7.

34. Coimbra IB, Pastor EH, Greve JMD, Puccinelli MLC, Fuller R, Cavalcanti FS, et al. Consenso brasileiro para o tratamento da osteoartrite (artrose). Rev Bras Reumatol 2002;42(6):371. 


\section{Anexos}

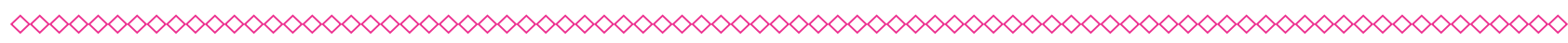

Tabela 1 - Comparação das variáveis entre as participantes do GI e GC, na avaliação.

\begin{tabular}{lccc} 
Variáveis & GI & GC & $P$ \\
Dor (pontuação de 0-10) & $7,43( \pm 1,8)$ & $7,92( \pm 1,8)$ & 0,582 \\
Função do joelho (pontuação de 0-100) & $30,6( \pm 17,4)$ & $27,23( \pm 14,4)$ & 0,585 \\
Levantar da cadeira (repetições) & $5,6( \pm 2)$ & $6,08( \pm 3,4)$ & 0,702 \\
Flexão de braço (repetições) & $10( \pm 3,5)$ & $12,08( \pm 5,8)$ & 0,275 \\
Sentar e alcançar os pés (cm) & $-13,8( \pm 15,6)$ & $-9,5( \pm 10)$ & 0,408 \\
Levantar e caminhar (s) & $10,2( \pm 8,1)$ & $9,2( \pm 4,7)$ & 0,695 \\
Alcançar as costas (cm) & $-21,5( \pm 11)$ & $-16,31( \pm 14,12)$ & 0,285 \\
Caminhada de $\mathbf{6}$ minutos $\mathbf{( m )}$ & $327,6( \pm 129)$ & $381( \pm 110)$ & 0,250 \\
\hline
\end{tabular}

(clique para voltar ao texto) 
Tabela 2 - Comparação das variáveis do estudo, entre as participantes do Grupo Intervenção (GI; $n=15$ ) e Grupo Controle (GC; $n=13$ ) e dentre as participantes dos grupos na avaliação e reavaliação.

\begin{tabular}{|c|c|c|c|}
\hline Variáveis & $\begin{array}{l}\text { Grupo intervenção } \\
\text { (GI) }\end{array}$ & $\begin{array}{l}\text { Grupo controle } \\
\text { (GC) }\end{array}$ & $p^{\S}$ \\
\hline \multicolumn{4}{|c|}{ Dor (pontuação de 0-10) } \\
\hline Avaliação & $7,43( \pm 1,8)$ & $7,92( \pm 1,8)$ & \\
\hline Reavaliação & $5,47( \pm 3,2)$ & $6,46( \pm 2)$ & 0,354 \\
\hline $\mathrm{p} ¥$ & $0,005^{*}$ & $0,013 *$ & \\
\hline \multicolumn{4}{|c|}{$\begin{array}{l}\text { Função do joelho } \\
\text { (pontuação de 0-100) }\end{array}$} \\
\hline Avaliação & $30,6( \pm 17,4)$ & $27,23( \pm 14,4)$ & \\
\hline Reavaliação & $48,20( \pm 19,7)$ & $30,62( \pm 12,3)$ & $0,009 *$ \\
\hline $\mathrm{p} ¥$ & $0,005^{\star}$ & 0,149 & \\
\hline \multicolumn{4}{|c|}{$\begin{array}{l}\text { Levantar da cadeira (repetições) } \\
\text { Força de MMII }\end{array}$} \\
\hline Avaliação & $5,6( \pm 2)$ & $6,08( \pm 3,4)$ & \\
\hline Reavaliação & $7,4( \pm 2,1)$ & $6,69( \pm 2,7)$ & 0,453 \\
\hline $\mathrm{p} ¥$ & $0,005^{\star}$ & 0,329 & \\
\hline \multicolumn{4}{|c|}{ Flexão de braço (repetições) } \\
\hline \multicolumn{4}{|c|}{ Força de MMS } \\
\hline Avaliação & $10( \pm 3,5)$ & $12,08( \pm 5,8)$ & \\
\hline Reavaliação & $14( \pm 3,3)$ & $12,62( \pm 3,9)$ & 0,302 \\
\hline $\mathrm{p} ¥$ & $0,001 *$ & 0,680 & \\
\hline \multicolumn{4}{|c|}{$\begin{array}{l}\text { Sentar e alcançar os pés }(\mathrm{cm}) \\
\text { Flexibilidade de MMII }\end{array}$} \\
\hline Avaliação & $-13,8( \pm 15,6)$ & $-9,5( \pm 10)$ & \\
\hline Reavaliação & $-8,8( \pm 16,9)$ & $-7,9( \pm 9,1)$ & 0,869 \\
\hline$p ¥$ & 0,138 & 0,668 & \\
\hline \multicolumn{4}{|c|}{$\begin{array}{l}\text { Levantar e caminhar (s) } \\
\text { Agilidade e Equilíbrio Din }\end{array}$} \\
\hline Avaliação & $10,2( \pm 8,1)$ & $9,2( \pm 4,7)$ & \\
\hline Reavaliação & $7,9( \pm 2,6)$ & $8,8( \pm 2,6)$ & 0,382 \\
\hline$p ¥$ & 0,152 & 0,627 & \\
\hline \multicolumn{4}{|c|}{$\begin{array}{l}\text { Alcançar as costas }(\mathrm{cm}) \\
\text { Flexibilidade de MMSS }\end{array}$} \\
\hline Avaliação & $-21,5( \pm 11)$ & $-16,31( \pm 14,12)$ & \\
\hline Reavaliação & $-23,4( \pm 9,6)$ & $-17,62( \pm 15,41)$ & 0,238 \\
\hline $\mathrm{p} ¥$ & 0,522 & 0,670 & \\
\hline \multicolumn{4}{|c|}{$\begin{array}{l}\text { Caminhada de } 6 \text { minutos (m) } \\
\text { Resistência Aeróbica }\end{array}$} \\
\hline Avaliação & $327,6( \pm 129)$ & $381( \pm 110)$ & \\
\hline Reavaliação & $382,2( \pm 115)$ & $368( \pm 113)$ & 0,747 \\
\hline $\mathrm{p} ¥$ & $0,000 *$ & 0,433 & \\
\hline
\end{tabular}


Figura 1 - Frequência do resultado final nominal de função do joelho na avaliação e reavaliação do GI e GC, obtidos por meio da aplicação do Questionário Específico para Sintomas do Joelho Lysholm Knee Scoring Scale.

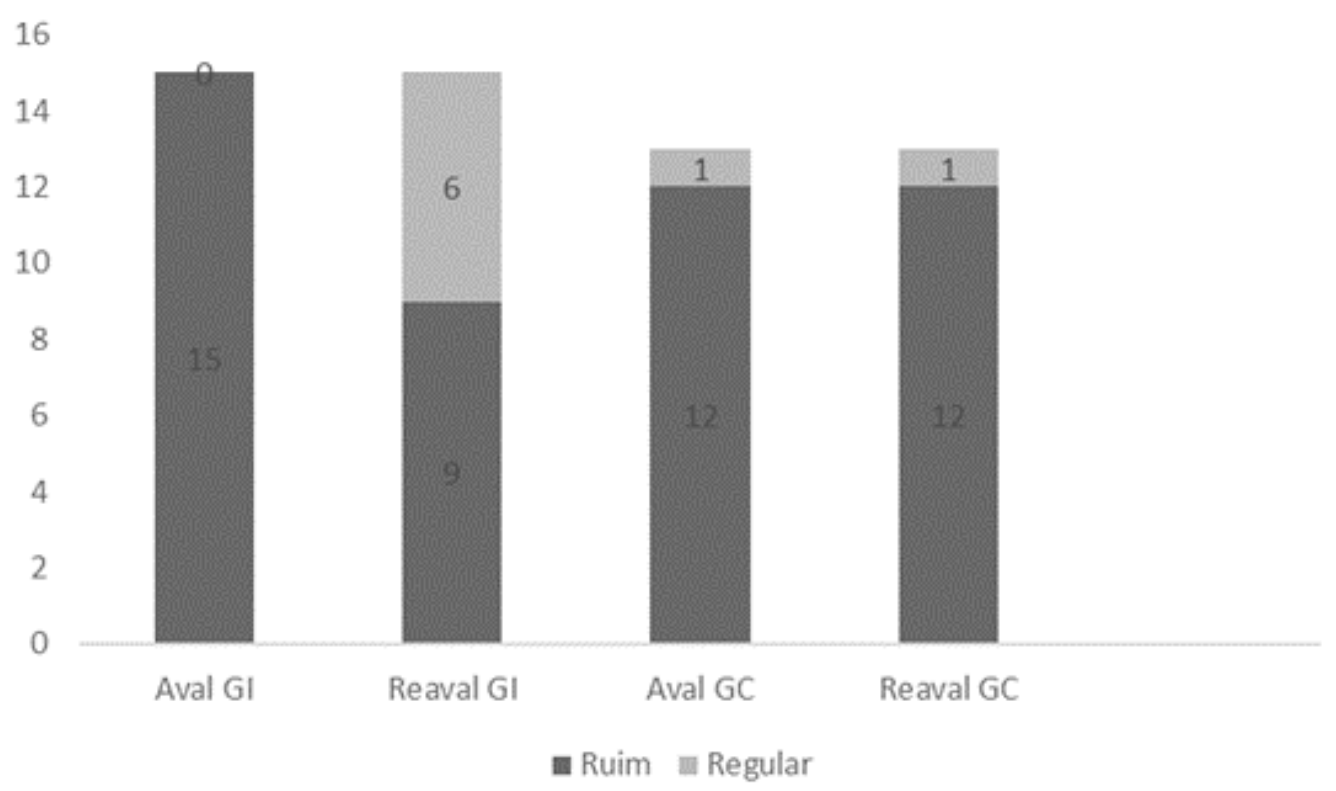

(clique para voltar ao texto)

Figura 2 - Frequência da intensidade da dor referida pelas participantes do GI e GC, na avaliação e reavaliação.

16

14

12

10

\section{8}

6

4

2

0

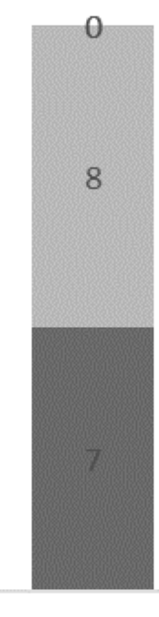

Aval GI

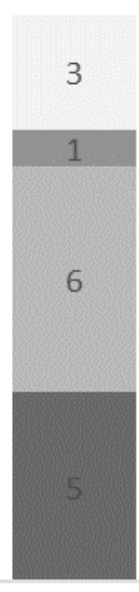

Reval GI

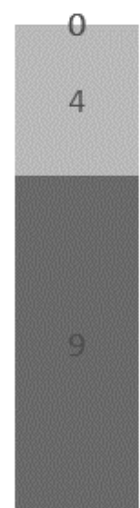

Aval GC

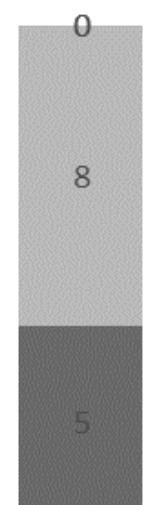

Reaval GC

- Intensa Moderada $\quad$ Leve Sem dor 